\title{
Distribution of different Skeletal pattern in Patients seeking Orthodontic Treatment in Mid-western Nepal
}

\author{
Dr Hemant Kumar Halwai, Dr Vanita Gautam, ${ }^{2}$ Dr Manju Pandey³ \\ 'Associate Professor, ${ }^{3 R}$ Resident, Dept of Orthodontics, ${ }^{2}$ Associate Professor, Dept of Conservative Dentistry \\ UCMS College of Dental Surgery, Bhairahawa, Nepal
}

Correspondence: Dr Hemant Kumar Halwai; Email: drhemanthalway@gmail.com

\section{ABSTRACT}

Objective: To determine the distribution of different skeletal pattern in orthodontic patients visiting a tertiary center in midwestern Nepal.

Materials \& Method: A sample of 200 lateral cephalograms of Nepali patients visiting UCMS-College of Dental Surgery, Bhairahawa was obtained. The jaw base relationship was assessed from lateral cephalograms using angular measurement (ANB angle). Among the total sample size; 74 (37\%) were males and 126 (63\%) were female. Age was divided into 3 groups: 8-11 years, 12-17 years, and 18-36 years. Pearson Chi square test was done to test the association between age group and distribution of skeletal jaw base relationship. All data were recorded and analyzed with SPSS software.

Result: The distribution of skeletal malocclusion according to ANB angle classification revealed that the most prevalent skeletal malocclusion was Class II skeletal jaw base relationship accounting up to $97(48.5 \%$ ), followed by Class I 87 (43.5\%) and Class III $16(8 \%)$. The study showed statistically significant difference between gender and distribution of skeletal jaw base relationship $(p<0.05)$. However, there was no significant difference between the age groups and distribution of skeletal jaw base relationship $(p<0.05)$.

Conclusion: Most common skeletal jaw base relationship in patients seeking orthodontic patients was Class II followed by Class I and Class III in a tertiary center of mid-western Nepal.

Keywords: anterio-posterior jaw base, cephalometric, skeletal jaw base relationship

\section{INTRODUCTION}

The prevalence of malocclusion has increased in recent decades, and it is considered one of the most common dental problems together with dental caries, gingival disease and dental fluorosis. Malocclusion patterns vary among different populations due to the variations in genetic and environmental influences. Planning of orthodontic treatment and allocation of the resources in a certain geographic location require baseline data on the prevalence of different types of malocclusion in that area.' A large number of studies on the prevalence of malocclusion in different populations have been published. $^{2}$

Angle's classification of malocclusion is based on the mesiodistal relationship between the upper and lower dental arches. Subsequent studies revealed that the dental arch relationship is largely affected by the facial skeleton upon which the teeth are invested. Bjork pointed out that the local changes confined to the teeth can exhibit limited effect on jaw discrepancy, whereas the facial or cranial configurations may produce marked effects. Thus, the relationships between craniofacial morphology and malocclusion have long been an interest to orthodontists. Studies have primarily investigated the relationship between the type of malocclusion and cranial base angle, vertical facial dimensions, and jaw sizes. However few studies have investigated the relationship between the antero-posterior dental arch and jaw-base relationships.

Angle's classification of molar relationship is probably the most widely used method for recording malocclusion. Steiners described skeletal anterio-posterior jaw-base relationship using Angle ANB formed by Nasion (N), Point $A$ and Point B. However various methods exhibit their own inherent weakness based on the variability of factors other than the jaw relationship itself. The aim of the present study was to evaluate the pattern of skeletal malocclusion using ANB angle in Nepali patients seeking orthodontic treatment in UCMS-College of Dental Surgery, Bhairahawa, Nepal. 
Table 1: Distribution of sample according to age group

\begin{tabular}{|c|c|c|c|}
\hline Age Group & Male & Female & Total \\
\hline $8-11$ years & $2(33.33 \%)$ & $4(66.66 \%)$ & $6(3.0 \%)$ \\
\hline $12-17$ years & $34(47.88 \%)$ & $37(52.11 \%)$ & $71(35.5 \%)$ \\
\hline $18-36$ years & $38(30.89 \%)$ & $85(69.10 \%)$ & $123(61.5 \%)$ \\
\hline
\end{tabular}

Table 2: Distribution of skeletal jaw base relationship in Male and Female

\begin{tabular}{|c|c|c|c|c|}
\hline Parameter & Male & Female & Total & p-Value \\
\hline Skeletal Class I & $26(29.88 \%)$ & $61(70.11 \%)$ & $87(43.5 \%)$ & \\
\cline { 1 - 3 } Skeletal Class II & $44(45.36 \%)$ & $53(54.63 \%)$ & $97(48.5 \%)$ & \multirow{2}{*}{$0.009 *$} \\
\hline Skeletal Class III & $4(25 \%)$ & $12(75 \%)$ & $16(8 \%)$ & \\
\hline
\end{tabular}

*Statistically significant at p-value $<0.05$

Table 3: Distribution of skeletal jaw base relationship in various age ranges

\begin{tabular}{|c|c|c|c|c|c|}
\hline Age group & Class I & Class II & Class III & Total & p-Value \\
\hline $8-11$ years & $2(33.33 \%)$ & $3(50 \%)$ & $1(16.66 \%)$ & $6(3.0 \%)$ & 0.390 \\
\hline $12-17$ years & $27(38.02 \%)$ & $37(52.11 \%)$ & $7(9.85 \%)$ & $71(35.5 \%)$ & (NS) \\
\hline $18-36$ years & $58(47.15 \%)$ & $57(46.34 \%)$ & $8(6.50 \%)$ & $123(61.5 \%)$ & \\
\hline
\end{tabular}

*Statistically significant at p-value $<0.05$

\section{MATERIALS AND METHOD}

The data collection for the study was done through the retrospective records of the patients seeking orthodontic treatment at USMS-College of Dental Surgery, Bhairahawa Nepal. Pretreatment Lateral cephalometric radiographs and orthodontic casts were obtained of the patient who visited the department. The inclusion criteria for the sample were: complete set of pretreatment records including study model, photographs, lateral cephalogram, with no history of previous orthodontic treatment.

Selected orthodontic casts were mounted in centric occlusion and were prepared with symmetric bases. The records of 200 pretreatment lateral cephalometric radiographs were collected. The lateral cephalometric radiographs were taken in natural head position on digital cephalogram (Vatech, Korea with PaX-Image capturing software). Careful evaluation of antero-posterior jaw base relationship was done with manual tracing on the basis of SNA, SNB and ANB angles by a single examiner. The jaw base relationships were categorized into three groups (skeletal Class I, II, III). The data were recorded on data collection sheet. All data were recorded and analyzed with SPSS software version 22. Frequency tables were made to determine the distribution of different jaw-base relationships (ANB angle).

\section{RESULT}

Out of the total 200 samples; 74 (37\%) were male, 126 (63\%) were female. The age range of the subjects was $8-36$ years with the mean of 18.74 years. Age category was divided into 3 groups: age 8-11 years, $12-17$ years, and $18-36$ years. Among them 3\% belonged to age group 8-11 years, $35.5 \%$ belonged to $12-17$ years, and $61.5 \%$ belonged to $18-36$ years. The study showed that, age group 18-36 years was the most to seek the orthodontic treatment (Table 1).

According to ANB classification; the most prevalent skeletal malocclusion was Class II skeletal jaw base relationship followed by Class I skeletal and Class III skeletal. The distribution of skeletal jaw base relationship among male, female and total samples is given in Table 2. Likewise, the distribution of skeletal jaw base relationship among the age groups is given in Table 3.

The study showed the association between gender and distribution of skeletal jaw base relationship $(p<0.05)$; however there was no association between age ranges and distribution of skeletal jaw base relationship $(p<0.05)$ (Table 2, 3).

\section{DISCUSSION}

The present study reports data regarding the prevalence of skeletal malocclusion using ANB angle measurement. It provides clinicians with an understanding of the most common types of malocclusion among the orthodontic patients visiting UCMS-College of Dental Surgery, Bhairahawa. Although many studies described the prevalence and types of malocclusion; some variability between their findings exist due to the varying methods and indices used to assess and record the occlusal 
relationships, age differences of the study populations, examiner subjectivity, specific objectives, and differing sample sizes. ${ }^{2-4}$

The results showed that the most common type of skeletal malocclusion determined by angular measurement (ANB) was skeletal Class II (48.5\%) followed by Class I (43.5\%) and Class III (8\%). It is because of the fact that, our sample was selected among the patients seeking orthodontic treatment and those individuals tend to present more of Class II skeletal problems than the general population. The sample comprising of orthodontic patients in terms of gender distribution and prevalence of skeletal jaw relationship is biased in nature. Thus the data of this orthodontic population cannot represent the Bhairahawa population and Mid-western region.

Various studies found various occurrences of skeletal jaw base relationships. Jones found that the most common skeletal malocclusion among Saudi orthodontic patients were Class I (46.4\%) followed by Class II (27.5\%) and Class III (26.1\%). Higher percentages of Class III cases was as a result of examining a small sample size that tend to be less representative. ${ }^{5}$ Al-Jundi and Riba showed the majority of Saudi Arabian patients with Class I skeletal (50.20\%), followed by Class II (40.98\%) and Class III (8.82\%). ${ }^{6}$ Farawana found Class I (74.5\%) skeletal relationship as the most predominant in Iraqi population; followed by Cass || (15\%) and Class III (10\%). ' Zhou et al reported Class I skeletal jaw base relationship in $69.3 \%$ followed by Class III in $16.3 \%$ and Class II in $14.4 \%$ among the Chinese sample. ${ }^{8}$

Bishara et $a^{9}$ described changes in the sagittal jaw relationship, comparing the ANB angle and Wits method. They demonstrated that the interpretation of the results with two forms of analyses is dependent on the geometrical errors inherent with the methods. They also suggested that the vertical development of the face alters ANB value. ${ }^{9}$ Evaluation of the sagittal jaw relationship can be expressed either as an angle, or as a linear measurement. While the angular analyses include the variations due to facial height, jaw prognathism, and jaw inclination; the Wits appraisal is very sensitive to changes in the inclination of the occlusal plane. The downward and backward rotation of the mandible might explain why some of the cases diagnosed as skeletal Class I with ANB measurement were actually Class III cases with rotated mandible that masked the true skeletal relationship. ${ }^{9}$ The same argument can be used to explain the classification of skeletal Class II relationship. The downward and backward rotation of the mandible made the diagnosis of some cases lean towards Class II according to the ANB reading. The type of malocclusion is an important factor that reflects patient's motivation to seek orthodontic treatment. Wilmot et al reported that patients with severe sagittal Class II malocclusion had a higher motivation for orthodontic treatment than Class III patients. ${ }^{10}$

\section{CONCLUSION}

According to our study which assessed pretreatment records of patients seeking orthodontic treatment at UCMS-College of Dental Surgery, the most common skeletal jaw-base relationship was Class II (48.5\%) followed by Class I (43.5\%) and Class III (8\%). Most of the number of patient seeking orthodontic treatment were female (63\%) and most of the patient seeking orthodontic treatment were of age group 18-36 (61.5\%).

\section{OJN}

\section{REFERENCES}

1. Bishara S. Textbook of Orthodontics. 1st ed. Philadelphia, Saunders; 2001.

2. Tang El, Wei SH. Recording and measuring malocclusion: A review of the literature. Am J Orthood Dentafac Orthop 1993; 103(4):344-51.

3. Al-Baalkhi K, Al-Zahrani A. The pattern of malocclusion in Saudi Arabian patients attending for orthodontic treatment at the College of Dentistry, Kind Saud University, Riyadh. Saudi Dent J. 1994; 6(3):138-44.

4. Al dress A. Pattern of Skeletal and dental malocclusion in Saudi orthodontic patient. Saudi Med J 2012; 33(3):315-20.

5. Jones WB. Malocclusion and facial types in a group of Saudi Arabia patients referred for orthodontic treatment: a preliminary study. Br J Orthod. 1987; 14(3):143-6.

6. Al-Jundi A, Riba H. Pattern of malocclusion in a sample of Orthodontic patients from a hospital in the Kingdom of Saudi Arabia. Savant J Med \& Medical Sci 2015; 1 (1):14-21.

7. Farawana NW. Malocclusion in Iraq. Quintessence Int. 1987; 18(2):153-7.

8. Zhou L, Mok CW; Hagg U, McGrath C, Bendeus M, Wu J. Anteroposterior dental arch and jaw-base relationships in a population sample. Angle Orthod 2008; 78(6):1023-9.

9. Bishara SE, Fahl JA, Peterson LC. Longitudinal changes in the ANB angle and Wits appraisal: clinical implications. Am J Orthod 1983: 84(2):133-9.

10. Wilmot JJ, Barber HD, Chou D G, Org Kw. Association between severity of dentofacial deformity and motivation for orthodonticorthognathic surgery treatment. Angle Orthod 1993; 63(4):283-8. 